\title{
Changes in Adherence to Oral Medications of People With Mental Illness During the Pandemic: Neuroticism and Conscientiousness Personality Traits Moderate the Effect of Medication Beliefs
}

Fabio Ferretti ( $\square$ ferrefa@unisi.it)

University of Siena https://orcid.org/0000-0001-8897-0965

\section{Arianna Goracci}

Department of Molecular Medicine, University of Siena, Siena

Pier Francesco Laurenzi

Department of Molecular Medicine, University of Siena, Siena

Rossella Centola

Azienda Ospedaliera Universitaria 'San Giovanni di Dio e Ruggi d'Aragona'

Irene Crecchi

Azienda Ospedaliera Universitaria Senese

Aldo De Luca

Instituto Sperimentale Italiano Lazzaro Spallanzani

Janette Monzillo

Scuola di Specializzazione in Farmacia Università di Siena

Omar Guidi

Scuola di Specializzazione in Farmacia Ospedaliera, University of Siena

\section{Giusy Sinigaglia}

Scuola di Specializzazione in Farmacia Ospedaliera, University of Siena

\section{Alessandro Cuomo}

Department of Molecular Medicine, University of Siena, Siena

Simone Bolognesi

Department of Molecular Medicine, University of Siena, Siena

\section{Valter Travagli}

Universita degli Studi di Siena Dipartimento di Biotecnologie Chimica e Farmacia

Andrea Fagiolini

Department of Molecular Medicine University of Siena, Siena

Anna Coluccia

University of Siena

Andrea Pozza

University of Siena 


\section{Research Article}

Keywords: Big Five personality, adherence, beliefs about medications, COVID-19 pandemic, mental disorders

Posted Date: March 2nd, 2022

DOl: https://doi.org/10.21203/rs.3.rs-775918/v1

License: (c) (1) This work is licensed under a Creative Commons Attribution 4.0 International License. Read Full License 


\section{Abstract}

Objectives. After the declaration of pandemic status in several countries, the continuity of face-to-face visits in psychiatric facilities has been delayed, or even interrupted to reduce viral spread. Little is known about the personality factors associated with medication beliefs and adherence amongst individuals with mental illness during COVID-19 pandemic. This longitudinal study explored whether the big-five personality traits prospectively moderate the effects of medication beliefs on changes in adherence during the pandemic for a group of outpatients with psychosis or bipolar disorder.

Results. Thirteen outpatients undergoing face-to-face follow-up assessments during the pandemic were included (41 observations overall). Participants had stronger concerns about their psychiatric medications rather than beliefs about their necessity, and adherence to medications was generally low. Participants with higher necessity beliefs had a better adherence to medications than those who perceived less their benefits. People with higher conscientiousness and neuroticism traits and more concerned about the medication side effects had a poorer adherence.

These preliminary data suggest the importance of a careful assessment of the adherence to medications amongst people with psychosis/bipolar disorder during the pandemic. Interventions aimed to improve adherence might focus on patients' medication beliefs and their conscientiousness and neuroticism personality traits.

\section{Introduction}

After the declaration of pandemic status in several countries, to reduce viral spread the continuity of faceto-face follow-up visits in psychiatric facilities has been delayed or interrupted (1). As a consequence of this change of psychiatric settings, reduced access to mental health services was one of the predictors of poor adherence to oral medications, which in turn increased the risk of symptom deterioration and relapse (2-3).

Regardless of the stressful effects of the pandemic, poor adherence to psychiatric medications such as antipsychotics and mood stabilizers, is a relevant phenomenon in people with mental illness as around $40 \%$ of people with psychosis or bipolar disorder show poor adherence, including discontinuation/intermittent assumption of medication. Such behaviour can negatively impact on the clinical outcomes, thus increasing the risk of recurrence of episodes (4).

Several conceptualizations have been developed to understand the factors that influence medication adherence, amongst the most important of which are the beliefs that patients hold about their medicines. According to the Necessity-Concerns Framework (5), these beliefs comprise the necessity of medicines in terms of potential for effectiveness but also concerns about possible adverse consequences. Based upon these beliefs, patients may decide not to continue with the prescribed medication if they perceive the necessity or the chances of success to be low or the costs (e.g., possible side effects) to be high. An 
increasing amount of data supports the validity of this model and suggests that poor adherence is associated with beliefs of high concern and low necessity (6).

The Big Five model of personality that integrates most of the evidence in personality psychology (7-8), assumes that individual differences in personality characteristics can be organized into five broad trait domains: Extraversion (extravert people experience high levels of happiness and life satisfaction), Agreeableness (individuals high in this trait are helpful, warm and emphatic), Conscientiousness (persons high in this trait tend to be well-organized, goal-directed and persistent), Neuroticism (persons high in this trait tend to experience strong levels of distress) and Openness (people high in openness have broad interests and seek experiences).

A relatively large number of studies investigated the relationship between the Big Five personality traits and medication adherence in patients with chronic medical conditions. Findings from a systematic review showed that amongst the Big Five factors, Neuroticism and Conscientiousness are negatively and positively associated with medication adherence, respectively (9).

Some authors explored the role of Big Five personality traits in medication adherence amongst people with psychiatric disorders, showing that only low Conscientiousness predicted a lower adherence (10). Other researchers hypothesized that the Big Five factors could moderate the effects of medication beliefs on adherence. In people with chronic diseases, Agreeableness, Conscientiousness and Neuroticism were associated with concerns about medication, and these concerns moderated the effects of these personality traits on adherence behaviour (11).

In summary, little is known about the factors associated with medication adherence amongst individuals with mental illness during the pandemic. Starting from the available evidence, the role of medication beliefs on adherence might be moderated by specific Big Five personality traits. This study explored whether the Big Five personality traits moderate the effects of medication beliefs on changes in adherence during the pandemic for a group of outpatients with mental illness.

\section{Methods}

\section{Eligibility criteria}

Participants were included if: they met the criteria for a primary diagnosis of psychotic/bipolar disorder according to the Diagnostic and Statistical Manual of Mental Disorders-fifth edition (DSM-5) (12); they were on antipsychotics and/or mood stabilizers; they were 18 years or older; and they signed informed consent. Participants were excluded if they had a neurological disorder, mental retardation and/or difficulty understanding written Italian.

\section{Measures}




\section{Revised Italian Version of the Ten-Item Personality Inventory (I-TIPI; 13)}

The I-TIPI is a revised version of the Ten-Item Personality Inventory (TIPI; 14), a questionnaire consisting of 10 items on a seven-point Likert scale that evaluates personality traits through five scales corresponding to the Big Five factors. It showed good reliability (13).

\section{Beliefs about Medicines Questionnaire (BMQ; 15)}

The BMQ is an 11-item questionnaire comprising two subscales assessing beliefs about medication prescribed for a condition, relating to perceptions about the personal need for medication (Necessity subscale) and concerns about potential negative effects from medication (Concerns subscale). The following are representative items for the Necessity and Concerns subscales, respectively: "My life would be impossible without these medications", "I sometimes worry about the long-term effects of these medications". The Italian validation showed good reliability (16).

\section{Morisky Medication Adherence Scale-8 item (MMAS-8; 17- 19)}

It is an 8-item questionnaire on a 5-point Likert scale that measures adherence to medications for a condition. The Italian validation showed reliability (20).

\section{Beck Depression Inventory II (BDI-II; 21)}

The BDI-II is a 21 -item questionnaire that rates the severity of depressive symptoms. Higher scores denote higher depression. The Italian version showed excellent reliability (22).

\section{Statistical analyses}

Owing to the longitudinal design, a generalized estimating equation (GEE) model was selected to examine the relationship between a dependent variable (medication adherence, measured by the MMAS8) and a set of predictors (beliefs about medication, BMQ; symptoms of depression, BDI-II; personality traits, I-TIPI). The difference between the BMQ-Necessity and BMQ-Concerns subscales ( $\triangle \mathrm{BMQ})$ was used as an independent variable. A number of models were estimated, including models with main effects and models with both main effects and interaction terms. The goodness of fit was assessed through the quasi-likelihood under independence model criterion (QIC) and corrected QIC (QICC), looking at the model that minimized these two indices. The assumptions of the final model were checked and the significance level was set at $p<0.05$. The analyses were performed using the Statistical Packages for Social Science (SPSS) v.25.

\section{Results}


Thirteen outpatients undergoing psychiatric face-to-face follow-up were included (sociodemographic/clinical features in Table 1). These subjects received a mean of 3 face-to-face assessments.

Table 1

Sociodemographic characteristics and clinical features of the sample $(n=$ 13)

\begin{tabular}{|c|c|c|}
\hline \multicolumn{2}{|l|}{ Age (years)* } & \multirow{2}{*}{$\begin{array}{l}\mathbf{5 7 . 7} \pm \mathbf{1 4 . 4} \\
8(61.5)\end{array}$} \\
\hline Gender** & Female & \\
\hline & Male & $5(38.5)$ \\
\hline \multirow[t]{3}{*}{ Marital status** } & Single & $2(16.7)$ \\
\hline & Married & $9(75.0)$ \\
\hline & Widowed & $1(8.3)$ \\
\hline \multirow[t]{2}{*}{ Occupational status** } & Employed & $4(30.8)$ \\
\hline & Unemployed & $9(69.2)$ \\
\hline \multirow[t]{2}{*}{ Education level** } & High school or lower & $7(58.3)$ \\
\hline & Degree or higher & $5(41.7)$ \\
\hline \multirow[t]{2}{*}{ Comorbid disorders** } & Yes & $5(38.5)$ \\
\hline & No & $8(61.5)$ \\
\hline \multirow[t]{3}{*}{ Type of psychiatric medications ${ }^{\star \star}$} & Antipsychotics & \\
\hline & Mood stabilizers & \\
\hline & Antidepressants & \\
\hline \multicolumn{2}{|c|}{ Duration of primary diagnosis (months)* } & $85.2 \pm 77.9$ \\
\hline \multicolumn{2}{|c|}{ Duration of psychiatric treatment (months)* } & $93.0 \pm 77.5$ \\
\hline \multirow[t]{2}{*}{ Previous hospitalizations } & Yes & $6(54.5)$ \\
\hline & No & $5(45.5)$ \\
\hline \multirow[t]{2}{*}{ Comorbid medical disorders** } & Yes & $8(61.5)$ \\
\hline & No & $5(38.5)$ \\
\hline
\end{tabular}


The scores of the questionnaires for all the measurements are summarized in Table 2. The two subscales of the BMQ showed that respondents had stronger concerns about their medication than beliefs about the necessity of these medical treatments; the difference between the two subscale scores $(\triangle \mathrm{BMQ})$ was -7 (IQ range $=6$ ), showing that patients' perceptions about medical treatments were mainly driven by their concerns. The degree of medication adherence, measured by the MMAS-8, was quite low: its median value was 3.5 (IQ range $=2$ ) and only one patient showed the maximum score of 6 , reflecting medium adherence. A mild level of symptoms of depression on the BDI-II was found (median $=14$; IQ range $=16$ ) but, taking into account all the measurements collected at the different time-points, $4 / 40(10.0 \%)$ revealed severe symptoms of depression and 9/40 (22.5\%) showed a moderate level of depression. Participants' personality traits are described through the I-TIPI score: this group of subjects was characterized by high levels of agreeableness, conscientiousness, and neuroticism but low levels of extraversion and openness. On comparing these findings with the population norms according to age and gender, $67.5 \%$ of the measurements related to the extraversion trait, $97.5 \%$ of the scores collected for the openness trait were below the norms and the scores for agreeableness, conscientiousness and neuroticism were frequently above the norms $(65 \%, 55 \%$ and $70 \%$ of the individual scores, respectively).

Table 2

Descriptive statistics of the BMQ, MMAS-8, BDI-II, and I-TIPI scores

\begin{tabular}{|c|c|c|c|c|c|c|}
\hline & Mean & SD & Median & IQ range & Min & Max \\
\hline BMQ Necessity & 11.4 & 3.8 & 10.5 & 6 & 5 & 18 \\
\hline BMQ Concerns & 18.3 & 5.2 & 18 & 7 & 10 & 30 \\
\hline$\triangle \mathrm{BMQ}$ (Necessity-Concerns) & -6.9 & 5.3 & -7 & 6 & -25 & 0 \\
\hline MMAS-8 & 3.5 & 1.2 & 3.0 & 2 & 1 & 6 \\
\hline BDI-II & 15.7 & 11.9 & 14.0 & 16 & 0 & 48 \\
\hline I-TIPI Extraversion & 3.6 & 1.5 & 3.5 & 2.6 & 1.5 & 6.5 \\
\hline I-TIPI Agreeableness & 5.5 & 1.0 & 6.0 & 1.0 & 3.5 & 7.0 \\
\hline I-TIPI Conscientiousness & 5.1 & 1.2 & 5.0 & 2.4 & 3.0 & 7.0 \\
\hline I-TIPI Neuroticism & 5.0 & 1.1 & 5.0 & 1.0 & 2.5 & 7.0 \\
\hline I-TIPI Openness & 3.5 & 0.8 & 3.5 & 1.4 & 2.0 & 5.0 \\
\hline \multicolumn{7}{|c|}{ Note. $\triangle \mathrm{BMQ}$ was obtained by the difference between BMQ Necessity and BMQ Concerns. } \\
\hline \multicolumn{7}{|c|}{$\begin{array}{l}\text { The MMAS-8 Scale, content, name, and trademarks are protected by US copyright and trademark } \\
\text { laws. Permission for use of the scale and its coding is required. A license agreement is available from } \\
\text { MMAR, LLC., Donald E. Morisky, ScD, ScM, MSPH, } 294 \text { Lindura Ct., USA; } \\
\text { donald.morisky@moriskyscale.com. }\end{array}$} \\
\hline
\end{tabular}


With the aim of exploring the relationship between the dependent variable, expressed by the degree of medication adherence, and its predictors (symptoms of depression, personality traits and beliefs about medicines), a series of GEE models were estimated, choosing the model that minimized both the QIC and QICC criteria. The final model included three predictors: the main effect of $\triangle B M Q$, and two interaction terms (I-TIPI Conscientiousness and I-TIPI Emotional Stability) with $\triangle B M Q$. The model's QIC and QICC were 49.7 and 45.5 , respectively, and the lack of multicollinearity was confirmed by comparison between Type I and Type III sum of squares estimation. The results of the model estimation are in Table 3 . All the parameters were significant. The positive sign of $\triangle B M Q$ showed that an increasing perception about the treatment necessity compared to concerns produced a stronger degree of medication adherence $(\beta=0.665, p=0.000)$. However, the model showed that some personality traits, such as conscientiousness and neuroticism, affected adherence to medications when they interacted with beliefs about medications: higher scores in neuroticism and conscientiousness resulted in a significant decrease in medication adherence (respectively: $\beta=-0.049, p=0.001 ; \beta=-0.065, p=0.004$ ).

Table 3

Results of the model estimation

\begin{tabular}{|llllll|}
\hline & $\boldsymbol{\beta}$ & SE & $95 \% \mathrm{Cl}$ & Wald $\chi^{2}$ & $\boldsymbol{p}$ \\
\hline Intercept & 4.165 & 0.461 & $(3.262-5.068)$ & $\chi^{2}{ }_{(1)}=81.749$ & 0.000 \\
\hline BBMQ & 0.665 & 0.173 & $(0.326-1.003)$ & $\chi^{2}{ }_{(1)}=14.827$ & 0.000 \\
\hline I-TIPI Conscientiousness · $\Delta \mathrm{BMQ}$ & -0.065 & 0.023 & $(-0.109$ to -0.021$)$ & $\chi^{2}{ }_{(1)}=8.329$ & 0.004 \\
\hline I-TIPI Neuroticism $\cdot \Delta \mathrm{BMQ}$ & -0.049 & 0.014 & $(-0.076$ to -0.021$)$ & $\chi^{2}{ }_{(1)}=11.856$ & 0.001 \\
\hline
\end{tabular}

\section{Discussion}

The results showed that, during the pandemic, people with mental illness have stronger concerns about their psychiatric medications than beliefs about the necessity of these treatments, and that adherence to medications is generally low in this population. The perceived necessity of the medications seems to have a more important role in adherence than perceived concerns: people with higher perceived necessity beliefs would have better adherence to medications than those who perceive their benefits as lower.

Interestingly, specific Big Five personality traits seem to moderate the relationship between medication beliefs and adherence; in particular, people with higher levels of conscientiousness and neuroticism traits and perceived concerns about the medication side effects seem to have poorer adherence. This appears to be partly consistent with the findings reported in samples with psychiatric or other chronic medical disorders assessed before the pandemic (9-11). The present findings expand current knowledge about the role of medication beliefs on adherence, highlighting the moderating effects of specific personality traits. The fact that high conscientiousness moderates the effect of perceived concerns seems to be slightly inconsistent with the literature and the Big Five model, which assumes that conscientiousness is 
a functional, goal-directed trait (8). An explanation might be that if people who endorse higher concerns about the side effects of their medicines also have a conscientiousness trait, they could have a persistent and goal-directed mental attitude towards a negative consideration of the side effects, which in turn could increase the likelihood of poor adherence. This explanation seems to be consistent with data highlighting the so-called downside of high levels of conscientiousness for psychological wellbeing (i.e., the effect of high levels of conscientiousness on low levels of wellbeing), as previously suggested (2324). In addition, high levels of conscientiousness might be associated with excessive attention focused on bodily signals typical of hypochondriac and/or obsessive-compulsive traits, which can generally be related to a catastrophic perception of side effects of medications (25-26). The role of conscientiousness traits influencing a greater perception of the side effects appears to be in line also with data showing that high levels of this trait are related to poorer understanding of the clinical information received from healthcare professionals (27).

Overall, these findings suggest the importance of careful assessment of adherence to psychiatric medications amongst people with mental illness during the pandemic. Interventions aimed to improve adherence to medications during the pandemic should focus on the beliefs that individuals endorse about the medicines they take. In addition, people with neuroticism and conscientiousness traits and high concerns about the medicines should be considered as a subgroup of patients potentially at risk of low adherence.

\section{Limitations}

Despite this study using a longitudinal design, the small sample size should be considered as a strong limitation that might have influenced the statistical power of the analyses and prevented further analysis. Another issue is the fact that we did not explore the effects of other variables, such as different types of medications, phases of the pandemic or number of visits with psychiatric professionals during the pandemic, which should be analysed in future research. In addition, the self-report measures used to assess medication adherence should be integrated with other sources of information, such as instruments completed by informal caregivers and biological markers. Another aspect that deserves future consideration is whether the effect of personality traits and medication beliefs on adherence can, in turn, predict a higher risk of relapse.

\section{Abbreviations}

BDI-II = Beck Depression Inventory-II

$\mathrm{BMQ}=$ Beliefs about Medicines Questionnaire

DSM-5 = Diagnostic and Statistical Manual of Mental Disorders-fifth edition

$\mathrm{GEE}=$ generalized estimating equation 
I-TIPI = Revised Italian Version of the Ten-Item Personality Inventory

MMAS-8 = Morisky Medication Adherence Scale-8 item

QIC = independence model criterion

$\mathrm{QICC}=$ corrected QIC

SPSS $=$ Statistical Packages for Social Science

TIPI = Ten-Item Personality Inventory

\section{Declarations}

\section{Ethics approval and consent to participate}

The study was approved by Comitato Etico Regionale per la Sperimentazione Clinica della Regione Toscana on $15^{\text {th }}$ July 2019 (approval code $n^{\circ}$ 15006). In accordance with the Helsinki Declaration, patients provided written informed consent to participate to the study.

\section{Consent for publication}

A MMAS Entitlement Certificate was requested and acquired from MMAR, LLC., Donald E. Morisky, ScD, ScM, MSPH, 294 Lindura Ct., USA; donald.morisky@moriskyscale.com. The entitlement certificate attached has been attached in the present submission as proof of license.

\section{Availability of data and material}

All data generated or analyzed during this study are included in this published article.

\section{Competing interests}

The authors declare that they have no competing interests.

\section{Funding}

No funding supported this study.

\section{Authors' contributions}

FF: designed the study, analyzed the data, wrote the research protocol, wrote the paper draft, edited the final version of the manuscript.

AG: designed the study, recruited the participants, and collected the data, wrote the paper draft.

PFL: designed the study, recruited the participants, and collected the data, wrote the paper draft. 
RC: designed the study, conducted the literature searches, wrote the paper draft.

IC: designed the study, conducted the literature searches, wrote the paper draft.

ADL: designed the study, conducted the literature searches, wrote the paper draft.

JM: designed the study, conducted the literature searches, wrote the paper draft.

OG: designed the study, conducted the literature searches, wrote the paper draft.

GS: designed the study, conducted the literature searches, wrote the paper draft.

AC: designed the study, conducted the literature searches, edited the final version of the manuscript.

SB: designed the study, conducted the literature searches, edited the final version of the manuscript.

AF: designed the study, wrote the research protocol, managed and monitored participants. recruitment and data collection, edited the final version of the manuscript.

AC: designed the study, wrote the research protocol, wrote the paper draft, edited the final version of the manuscript.

AP: designed the study, conducted the literature searches, wrote the research protocol, wrote the paper draft, edited the final version of the manuscript.

\section{Acknowledgements}

The MMAS-8 Scale, content, name, and trademarks are protected by US copyright and trademark laws. Permission for use of the scale and its coding is required. A license agreement is available from MMAR, LLC., Donald E. Morisky, ScD, ScM, MSPH, 294 Lindura Ct., USA; donald.morisky@moriskyscale.com.

\section{References}

1. Fagiolini A, Cuomo A, Frank E. COVID-19 diary from a psychiatry department in Italy. J Clin Psychiatry. 2020;81:13357.

2. Chatterjee SS, Malathesh Barikar C, Mukherjee A. Impact of COVID-19 pandemic on pre-existing mental health problems. Asian J Psychiatr. 2020;51:102071.

3. Kølbæk P, Jefsen $\mathrm{OH}$, Speed M, Østergaard SD. Mental health of patients with mental illness during the COVID-19 pandemic lockdown: A questionnaire-based survey weighted for attrition. 2021;MedRxiv.

4. Sajatovic M, Valenstein M, Blow FC, Ganoczy D, Ignacio RV. Treatment adherence with antipsychotic medications in bipolar disorder. Bipolar Disord. 2006;8:232-241. 
5. Horne R, Chapman SC, Parham R, Freemantle N, Forbes A, Cooper V. Understanding patients' adherence-related beliefs about medicines prescribed for long-term conditions: A meta-analytic review of the Necessity-Concerns Framework. PloS One. 2013;8:e80633.

6. De las Cuevas $C$, Betancort M, de Leon J. The necessity-concern framework in the assessment of treatment adherence of psychiatric patients and the role of polypharmacy in a Spanish sample. Hum Psychopharmacol. 2020;35:e2721.

7. McCrae RR, Costa PT Jr. Personality in adulthood: A five-factor theory perspective (2nd ed.). New York: Guilford, 2003.

8. Graham EK, Weston SJ, Gerstorf D, Yoneda TB, Booth T, Beam CR, ... Mroczek DK. Trajectories of big five personality traits: A coordinated analysis of 16 longitudinal samples. Eur J Pers. 2020;34:301321.

9. Driesenaar J. Beliefs and Adherence regarding Inhaled Corticosteroids. Gildeprint - Enschede, 2018.

10. Strickland JC, Stoops WW., Kincer MA, Rush CR. The impact of financial strain on medication nonadherence: Influence of psychiatric medication use. Psychiatry Res. 2019;271:389-395.

11. Axelsson M, Brink, E, Lundgren, J, Lötvall, J. The influence of personality traits on reported adherence to medication in individuals with chronic disease: An epidemiological study in West Sweden. PloS One. 2011;6:e18241.

12. American Psychiatric Association. Diagnostic and statistical manual of mental disorders. 5th ed. Washington, DC: American Psychiatric Association, 2013.

13. Chiorri C, Bracco F, Piccinno T, Modafferi C, Battini V. Psychometric properties of a revised version of the Ten Item Personality Inventory. Eur J Psychol Assess. 2015;31:109-119.

14. Gosling SD, Rentfrow PJ, Swann WB Jr. A very brief measure of the Big-Five personality domains. J Res Pers. 2003;37:504-528.

15. Horne R, Weinman J, Hankins M. The Beliefs about Medicines Questionnaire: The development and evaluation of a new method for assessing the cognitive representation of medication. Psychol Health. 1999;14:1-24.

16. Argentero P, Torchio E, Tibaldi G, Horne R, Clatworthy J, Munizza C. The beliefs about drug treatments. The Italian version of the BMQ (the beliefs about medicines questionnaire): its validity and applicability. Epidemiol Psychiatr Sci. 2010;19:86-92.

17. Morisky DE, Ang A, Krousel-Wood M, Ward H. Predictive Validity of Medication Adherence Measure in an Outpatient Setting. J Clin Hypertens. 2008;10:348-354.

18. Berlowitz DR, Foy CG, Kazis LE, Bolin L, Conroy LB, Fitzpatrick P, et al. for the SPRINT Study Research Group. Impact of Intensive Blood Pressure Therapy on Patient-Reported Outcomes: Outcomes Results from the SPRINT Study. N Engl J Med. 2017;377:733-44.

19. Bress AP, Bellows BK, King J, Hess R, Beddhu S, Zhang Z, et al, for the SPRINT Research Group and the SPRINT Economics and Health Related Quality of Life Subcommittee. Cost-Effectiveness of Intensive versus Standard Blood Pressure Control. N Engl J Med. 2017;77:745-55. 
20. Fabbrini G, Abbruzzese G, Barone P, Antonini A, Tinazzi M, Castegnaro G, ... REASON study group. Adherence to anti-Parkinson drug therapy in the "REASON" sample of Italian patients with Parkinson's disease: the linguistic validation of the Italian version of the "Morisky Medical Adherence Scale-8 items". Neurol Sci. 2013;34:2015-2022.

21. Beck AT, Steer RA, Ball R, Ranieri WF. Comparison of Beck Depression Inventories-IA and-II in psychiatric outpatients. J Pers Assess. 1996;67:588-597.

22. Sica C, Ghisi M. The Italian versions of the Beck Anxiety Inventory and the Beck Depression Inventory-II: Psychometric properties and discriminant power. In MA Lange (Ed.), Leading-edge psychological tests and testing research, pp. 27-50. Nova Science Publishers, 2007.

23. Boyce CJ, Wood AM, Brown GD. The dark side of conscientiousness: Conscientious people experience greater drops in life satisfaction following unemployment. J Res Pers. 2010;44:535-9.

24. Carter NT, Guan L, Maples JL, Williamson RL, Miller JD. The downsides of extreme conscientiousness for psychological well-being: The role of obsessive compulsive tendencies. $J$ Pers. 2016;84:510-522.

25. Cox BJ, Borger SC, Asmundson GJ, Taylor S. Dimensions of hypochondriasis and the five-factor model of personality. Pers Individ Diff. 2000;29:99-108.

26. Sugarman MA, Kirsch I, Huppert JD. Obsessive-compulsive disorder has a reduced placebo (and antidepressant) response compared to other anxiety disorders: a meta-analysis. J Affect Disord. 2017;218:217-226.

27. Ferguson E, Semper H, Yates J, Fitzgerald JE, Skatova A, James D. The 'dark side'and 'bright side'of personality: when too much conscientiousness and too little anxiety are detrimental with respect to the acquisition of medical knowledge and skill. PloS One. 2014;9:e88606. 